\title{
El resurgimiento de la sociedad civil: fundamentos para una filosofía de la solidaridad
}

The Resurgence of Civil Society: Foundations for a Philosophy of Solidarity

\author{
Romel Hernández ${ }^{l}$ \\ Universidad Cooperativa de Colombia - Pasto \\ Mauricio Chamorro ${ }^{2}$ \\ Universidad Cooperativa de Colombia - Pasto \\ Colombia
}

\section{Resumen}

El siguiente artículo tiene como objetivo reflexionar sobre el punto de encuentro de dos discusiones teóricas en torno a la dicotomía entre sociedad civil y Estado. Hay una importante discusión según la cual, en la actualidad, estamos frente a un "resurgimiento de la sociedad civil", caracterizado por la reafirmación de los valores del autogobierno, la expansión de la subjetividad, del comunitarismo y de las organizaciones autónomas de los intereses y los modos de vida. El artículo se elabora mediante una búsqueda y un uso de teorías que permitieron una discusión teórica sobre el tema y los conceptos desarrollados. De lo anterior se obtiene que el discurso solidario, hoy, crea tendencias en la economía, la política y la filosofía. Estas tendencias ya no son simples discursos al margen, sino que se muestran como dominantes y van más allá de las sencillas relaciones humanas, buscando la conservación del medio ambiente y la creación de unos lazos de armonía con otros seres vivos que habitan con nosotros este planeta. La

1 Doctorando en Ciencias Filosóficas, Magister en Filosofía. Profesor-investigador de la Facultad de Derecho de la Universidad Cooperativa de Colombia - Pasto. Correo electrónico: romel.hernandez@campusucc.edu.co

2 Magister en Antropología, Especialista en Estudios Latinoamericanos. Profesor-investigador de la Facultad de Derecho de la Universidad Cooperativa de Colombia - Pasto. Correo electrónico: alvarom. chamorro@campusucc.edu.co 
solidaridad, hoy más que nunca, se torna importante y es fundamental abordarla, estudiarla, darle al discurso que la contiene toda la difusión necesaria, a través de las tendencias económicas, políticas y filosóficas; pero, más importante aún que su difusión en este tiempo, es realmente esencial entender los motivos por los cuales actualmente la solidaridad tiene tanta trascendencia. Por ende, la discusión se da en torno a la preocupación de que actualmente existe una sociedad en búsqueda de encerrarse en su esfera privada para no ser molestados; el problema que surge de ahí, y por el cual los griegos no sufrían, es cómo hacer posible que ese Estado adquiera legitimidad y no se atomice, no se desarticule.

Palabras clave: sociedad civil; Estado; filosofía de la solidaridad.

\begin{abstract}
The following article aims to reflect on the meeting point of two theoretical discussions on the dichotomy between civil society and the State. There is an important discussion according to which, at present, we are facing a "resurgence of civil society," characterized by the reaffirmation of the values of self-government, the expansion of subjectivity, communitarianism and autonomous organizations of interests and ways of life. The article is written by means of a search and use of theories that allowed a theoretical discussion on the topic and the developed concepts. It follows from what was previously stated that, today, solidarity discourse creates trends in economics, politics, and philosophy. These tendencies are no longer simple isolated discourses but seem to be dominant and go beyond simple human relationships, seeking the preservation of the environment and the creation of bonds of harmony with other living beings that live with us on this planet. Solidarity, today more than ever, becomes important and it is fundamental to approach it, to study it, to give to the speech that contains it all the necessary diffusion, through the economic, political and philosophical tendencies; but, even more important than its diffusion in this time, it is really essential to understand the reasons why nowadays solidarity has so much transcendence. Therefore, the discussion revolves around the concern that today there is a society seeking to lock itself into its private sphere so as not to be disturbed. The problem arising from there, and for which the Greeks did not suffer, is how to make it possible for that State to acquire legitimacy and not to atomize, not to be disarticulated.
\end{abstract}

Keywords: civil society; state; philosophy of solidarity.

\title{
Introducción
}

La discusión teórica suscitada a partir de la contraposición entre la sociedad civil y el Estado es abordada desde dos posturas. La primera reconoce como una realidad esta dicotomía y sustenta su postura en que la acción estatal es un enemigo potencial de la libertad individual y de las condiciones para su ejercicio. Estos 
son los postulados de Karl Marx, para quien el término de sociedad civil apareció en el siglo XVIII, cuando ya las relaciones de propiedad se habían desprendido de los marcos de la comunidad antigua y medieval. La sociedad civil en cuanto tal solo se desarrolla con la burguesía; sin embargo, la organización social que se desarrolla basándose en la producción y el intercambio, así como forma en todas las épocas la base del Estado y de toda superestructura idealista, se ha designado siempre, invariablemente, con el mismo nombre (Marx, 1968).

La segunda postura considera que dicha separación es teóricamente insostenible, por cuanto al Estado le ha correspondido un papel muy activo y decisivo en la creación de condiciones institucionales y jurídicas para la expansión del mercado, característica indudable de la sociedad civil. Así, como afirma Boaventura de Sousa Santos, el siglo XIX inglés dio demostración de un incremento de legislación sobre la política económica y social, y también sobre la aparición de una amalgama de nuevas instituciones estatales como la "Factory Inspectorate", el "Poor Law Board", el "General Board Health", etc. "Esto implica que las políticas del laissez faire fueron aplicadas, en gran medida, a través de una activa intervención estatal. En otras palabras, el Estado tuvo que intervenir para no intervenir" (1998, p. 142).

Pese a esta discusión, y como punto de encuentro entre las dos posturas, encontramos que en la actualidad estamos frente a un "resurgimiento de la sociedad civil", que se caracteriza por la reafirmación de los valores del autogobierno, la expansión de la subjetividad, del comunitarismo y de las organizaciones autónomas de los intereses y los modos de vida. En este sentido, la solidaridad es vista como una manifestación del "resurgimiento de la sociedad civil" y en ella descansa su núcleo genuino. Sin embargo, en la actualidad el discurso solidario se hace más explícito en autores contemporáneos, siempre atado a construcciones teóricas de justicia; así resulta interesante reflexionar sobre este concepto tanto desde la historia como desde las diferentes posiciones filosóficas.

\section{La noción de solidaridad: juicio retrospectivo en la filosofía}

Si se hace un recorrido breve por el pensamiento griego, se encontrará que en él no existía ni la más mínima preocupación por lo que hoy se denomina solidaridad. En la República de Platón, el pensamiento central de sus diálogos es la justicia; intentar definirla se vuelve para los griegos un problema fundamental, pues la cohesión social griega, que entendía como iguales a los griegos con derechos y deberes para con el Estado (ciudadanos), era el eje cardinal de la ciudad o polis. No hay entre los griegos, o en el pensamiento griego, ningún texto que hable de 
forma manifiesta sobre lo que es la solidaridad. Pero, aunque no exista ningún escrito explícito sobre solidaridad, Platón pone, en sus diálogos, a hablar a su maestro Sócrates de la amistad y el amor (Fredo del amor y Lysis de la amistad). Son los dos diálogos escritos por Platón que hacen referencia a la solidaridad de manera indirecta. En ellos, la solidaridad no pasa de ser vista como la relación con el otro de forma afectiva. Pero es posible decir que Sócrates intenta darle al amor y a la amistad una explicación racional ontológica con la que se pueden definir. Y si se acepta esa explicación de amor y amistad que hay en Sócrates, es posible decir que, en dichos escritos, se encuentra una forma de explicar cómo surge la solidaridad.

En realidad no era la sociedad griega la interesada en hablar de la solidaridad; para ellas, como ya se dijo, es la justicia su columna vertebral, la justicia como sentido de la vida y, por lo tanto, como cohesión entre los hombres. El ser griegos los llevaba a sentirse capaces de ser justos entres sí (Werner, 1966). Fue el pensamiento griego que se difundía entre todos los ciudadanos lo que hacía posible una acción recíproca entre ellos, de ahí que la educación tenga como objetivo no solo formar individuos, sino formar ciudadanos capaces de participar en lo público (Werner, 1966). No había entre los griegos motivos por los cuales la solidaridad sea un tema inquietante; ni siquiera lo fue en momentos difíciles como en las guerras del Peloponeso o las guerras médicas.

Los griegos, si bien tenían una noción de solidaridad, no pasaba de ser entendida en los términos comunes de polis, en definitiva, no era una preocupación para ellos, pues no existía en su época una condición humana superior a la de ciudadano, ese que era la máxima forma de medición de los hombres y el árbitro que impartía tal medición era el Estado. El aporte que le hacen los griegos a la solidaridad es mínimo, no tienen motivos para pensar en esos temas y lo mismo pasó con el pensamiento de la Edad Media. El pensamiento utópico que comienza a romper con el obscurantismo de la Edad Media y le da origen al Renacimiento sienta las bases de una posible preocupación por la solidaridad, pues se constituye en un modelo moral de sociedad que intenta promover valores de integración humana. En los países de miel y mermelada que se pintaban en las diversas utopías, entre ellas las escritas por Tomás Moro o Campanela, el propósito era despertar un sentido de compasión y misericordia para con quienes sufrían y se sentían desgraciados por las condiciones sociales de este mundo (Pardo, 1990). Lo que se pretendía en esos modelos sociales utópicos era despertar una solidaridad entre los hombres; hacer que todos soñaran con un mundo mejor. Pero no había, en dichas invenciones utópicas, una explicación de la solidaridad. En el libro de Isaac Pardo, titulado Fuegos bajo el agua: la invención de utopía, es posible encontrar, 
bajo todo el recorrido que él hace sobre los discursos utópicos, elementos que sirvan para explicar por qué es hoy tan importante la solidaridad, pues si la utopía pretende despertar solidaridad entre los hombres debe hacerlo porque ve y entiende un problema que la hace necesaria.

Esa preocupación por la solidaridad que inicia en el Renacimiento tiene su continuación en el socialismo utópico. Es probablemente en ese momento cuando aparece por primera vez una preocupación, de manera inconsciente, de por qué es tan importante la solidaridad. Los socialistas utópicos, que querían cambiar el orden social-económico de competencia y ganancia por uno de reciprocidad y respeto por los otros, hablan de un principio de igualdad y de humanidad, apegados a la visión católica de humanidad en cuanto todos somos hijos de Dios, que llevaría a que cada hombre sea solidario con los otros, sin importar las circunstancias en las que esté.

Si bien, estos pensadores conocidos como socialistas utópicos ponen elementos que sirven para comprender los motivos por los cuales surge la preocupación por la solidaridad en la época moderna, no se puede, a partir de ellos, obtener una explicación concreta desde sus reflexiones. Realmente es con pensadores como Habermas, Rawls, Van Parijs Phillippe y algunos posmodernos como Lyotar o Levinas, cuando se puede apreciar el por qué la solidaridad tiene tanta importancia hoy en día. A diferencia de los griegos, para quienes las discusiones sobre la justicia estaban en tratar de definirla, Habermas y Rawls tienen una preocupación distinta, en realidad buscan el intento de legitimar la justicia, darle un sentido a esta. Para eso, acuden a la solidaridad, pero no hablan de ella como un discurso más o uno secundario en la vida social, no. Realmente, pretenden entender la solidaridad como un repotenciar de la justicia en una sociedad atomizada, fragmentada, que cobija a seres humanos diversos. Por ello, la pregunta fundamental de Rawls, a la que trata de responder en toda su obra titulada Teoría de la justicia, es: ¿Cómo es posible que la reunión de un conjunto de sujetos libres, racionales, razonablemente informados, con principios morales y motivados por un egoísmo más bien moderado, pueda levantar una sociedad inspirada en principios imparciales de justicia? (1971).

La justicia en Rawls es, cómo lo fue para los griegos, el elemento central de la discusión, pero en él no se trata de definirla sobre principios de igualdad, sino sobre la realidad de lo diverso. Los griegos no se preocupaban por la solidaridad, porque para ellos esta se encontraba presupuesta en cuanto la entendían a partir de la igualdad; de ahí que la justicia, según su pararecer, era un hecho entre griegos ciudadanos de un Estado, pero que debía definirse para ellos no para los otros. 
Rawls tiene la preocupación de legitimar la justicia para un mundo globalizado y por esta razón no puede argumentar que la justicia se da entre iguales, sino entre diferentes, pero para que opere, para que ella tenga validez, es necesario atarla con el cordón de la solidaridad.

Buscando legitimar la justicia, con casi el mismo objetivo de Rawls, Habermas acude a la democracia deliberativa. El objetivo fundamental de la democracia deliberativa que acuñó el filósofo Joseph M. Bessette es hacer posible la acción comunicativa. Partir del lenguaje como el elemento de interacción social que posibilita el entendimiento de los otros es lo que Habermas hace en Teoría de la acción comunicativa. Pero la comunicación o la posibilidad de interactuar a través del lenguaje debe llevarnos a una acción práctica, más allá del simple hablar. Por lo tanto, la organización política y en especial la democracia deben estar regidas por una amplia discusión, no debe ser solo el acto del sufragio el que haga posible la participación en la democracia. Al contrario, es preciso que la posibilidad de discutir y llegar a un consenso que trascienda el sufragio sea lo que permita la participación de los ciudadanos en las decisiones estatales.

El debate y el consenso de la plaza pública es lo que posibilita la democracia deliberativa (Habermas, 1998). Esa hace posible que la justicia tenga legitimidad, que las decisiones tomadas a través de la discusión tengan aceptación, pero al igual que Rawls, acude a cuestiones morales que involucran la solidaridad. Ahora bien, estos dos filósofos que acuden a la solidaridad lo hacen de tal forma que se convierte en un elemento necesario para la cohesión social, pues, al establecer ya no un principio de igualdad entre todos, sino un vínculo igual para todos que posibilita la comunicación y, por ende, el entendimiento, no se presupone a los hombres como iguales, sino que, en contraste, es su diferencia lo que se trata de resguardar. Tal diferencia debe tener algo que dé inicio a toda la democracia deliberativa, y ese principio es la solidaridad (Salvat, 2007).

Desde una orilla distinta a la que se ubican Habermas y Rawls, desde el marxismo analítico, Van Parijs Philippe acude al dilema entre economía o ética, inclinándose hacia el lado de la ética, al poner el acento sobre la solidaridad. Lo que Van Parijs P. se propone es hacer posible que las condiciones materiales de los hombres, en especial de los menos favorecidos, les permitan recobrar su dignidad para que les sea posible actuar en sociedad. La idea de una renta básica en Philippe contiene la noción esencial de solidaridad, pues esta acción estaría mediada por el Estado, en tanto sería él el que la proveería, pero la deduciría de las ganancias de aquellos que tienen un capital considerable. La renta básica es de carácter incondicional, pues quienes la reciben no tendrían que cumplir ninguna condición, 
porque el Estado tendría como deber garantizar que todos los ciudadanos tienen ingresos suficientes para satisfacer sus necesidades básicas (Van Parijs, 2004).

Para Van Parijs, se hace necesario refundar la solidaridad, porque no puede ser entendida solo en la justicia (Van Parijs, 1996). La solidaridad debe estar en la economía y de forma global (Van Parijs, 2002). Esto lo sostiene el filósofo porque la desigualdad nacional tiene las mismas causas que las desigualdades mundiales, pero a estas hay que agregarles y tener en cuenta las diferencias innatas de los hombres; por ello, en su libro sobre La diversidad cultural frente a la solidaridad económica, plantea que no se puede sostener una solidaridad nacional, es necesario una mundial que involucre aspectos de la economía y la justicia que establezcan unas generalidades entre las cuales sea posible la diversidad. Para Van Parijs la solidaridad económica, a diferencia de Habermas y Rawls, es la base de todo su andamiaje discursivo.

Hay también que, resaltar aquí que autores enmarcados en el movimiento denominado posmoderno, como Jena François Lyotar y Emanuel Levinas, hacen referencia a la solidaridad de manera un tanto específica, pero no apelando a la justicia o a la economía, sino desde una ángulo que abarca la crítica histórica como ha sido vista en la modernidad. En otras palabras, para ellos la historia como teleología no puede seguir teniendo sentido y por eso hablan de su fragmentación en historias posibles. En esta concepción del acontecer histórico, dichos autores hacen referencia a momentos especiales en los cuales, al parecer, se rompe la linealidad histórica y se construye momentos de ruptura. Para Lyotar, ese momento característico es mayo del 68, cuando lo que hace posible la construcción de una línea de fuga histórica es El entusiasmo, su libro referido a estos temas, porque de acuerdo con él, el actuar que hace posible este acontecimiento, el cual rompe con el paradigma del poder centrado en el Estado, se da precisamente por el incremento de un entusiasmo generalizado que se manifiesta en la gente. Tal entusiasmo contiene algo que hoy en día es la solidaridad, porque en $E l$ entusiasmo la adhesión a la causa común es espontánea, sin influencia de propaganda ni apegada a los grandes medios de comunicación.

Siguiendo esa línea de discusión en la posmodernidad, Emanuel Levinas, en su libro titulado La huella del otro, intenta analizar los acontecimientos sucedidos en la Alemania Nazi, no de forma descriptiva ni a manera de una narración histórica, sino a partir de una conceptualización filosófica que le permita entender el fenómeno de la relación de los hombres como individuales con los otros sujetos cuyas pretensiones también son individuales. Levinas hace una ruptura con la mirada de la filosofía moderna en cuanto a que esta parte del objeto como eje central de 
la relación; la dinámica de interacción sujeto-objeto prevalece en la modernidad, en especial en la modernidad científica. Para Levinas, de lo que se trata no es de conocer al otro como un objeto de estudio, ni como un sujeto-objeto que me permite conocerme a mí mismo, sino partir del otro como sujeto para poder entender realmente al otro. Dicho de forma más clara, no se trata de entender al otro en el sentido lato de la palabra, pues por lo general entender al otro es traducir a nuestras palabras lo que él es, para entenderlo como nosotros lo entendemos. Lo que Levinas quiere es entender al otro, tal cual el otro se muestra o quiere ser visto. Esta dinámica de interacción requiere una forma de relacionarse especial para una sociedad diversa y plural, que no parta de la igualdad, del lenguaje ni de las clases sociales, sino de una absoluta diferencia. Pero, para que haya necesidad de entender al otro como diferente, se hace fundamental presuponer una acción solidaria con él. No puede haber interés por lo otro diferente, si no hay, de antemano, una relación con este; esa relación es la solidaridad. Levinas no se refiere de forma explícita a esta última, pero la presupone.

\section{Sobre la importancia de una filosofía de la solidaridad}

El desarrollo del pensamiento económico, político y, en especial, el filosófico tuvo como eje central de discusión, durante mucho tiempo, la libertad y la igualdad. Así, en la Época Clásica, los diálogos de Platón y los escritos de Aristóteles remiten siempre a intenciones casi imposibles, que tratan de definir aquello que es la justicia, pero una siempre amparada por la libertad y la igualdad, para referirse solo a su carácter redistributivo (Young, 2000).

De la misma forma sucedió en la Edad Media y en la Época Moderna, cuando la presencia de jerarquías sociales impedía un reconocimiento generalizado (Honneth, 1997) que dificultaba la aparición de un discurso solidario. Sin embargo, con el nacimiento de la Ilustración que gesta el proyecto moderno, se pondrá en la Modernidad, al lado de la igualdad y la libertad, la fraternidad como un principio esencial de convivencia entre los seres humanos. Esto se hace explícito cuando la Revolución francesa proclama "Libertad, igualdad y fraternidad" como pilares inamovibles del proyecto burgués que se materializa en los derechos del hombre y del ciudadano.

Ese aparecer de la fraternidad como principio esencial de los derechos del hombre y del ciudadano, bases de los derechos humanos, no despierta, en los discursos académicos y políticos, gran apego por aquello que se conoce como solidaridad, seguían siendo la libertad y la igualdad los motivos de discusión constante (Mill, 1859). Aunque la Modernidad se caracteriza por darle grandes herramientas de 
pensamiento al discurso político liberal, representado en Hobbes, Locke y Hume, se comienza a gestar, en el lado de los arrabales franceses, el pensamiento socialista utópico que, con la pretensión de rescatar los ideales de la revolución traicionada (Revolución francesa), vuelve a darle importancia a la fraternidad. El socialismo utópico, apartándose de la visión individualista liberal, puso el acento sobre la condición social de los hombres y trató de rescatar la solidaridad como un valor humano innato. Sin embargo, para los socialistas utópicos, que apelaban a acciones pedagógicas para despertar el sentir social interno de los hombres, el surgimiento del socialismo científico estructurado por Marx opacó su discurso solidario de carácter pedagógico como alternativa frente a la naciente sociedad de mercado (Cole, 1957). Con Marx, tanto en el campo de la economía como en el de la política y filosofía, el discurso solidario volvió a jugar un papel secundario, se mantuvo la preocupación por la libertad y la igualdad. Los discursos economicistas que se polarizaban entre economía planificada y sociedades de mercado no dieron espacio para intentar el rescate del discurso solidario de forma pura, sin ligarlo a ninguna tendencia política o ideológica; la solidaridad volvía ser un apéndice que solo era posible después de ciertas transformaciones económicas.

Después de comenzar a desmoronarse la Modernidad y a darle paso a aquello que se denomina la Condición Posmoderna (Lyotar, 2000), algunos autores han retomado el discurso solidario y lo han puesto nuevamente en escena como el único elemento que hará posible restablecer la credibilidad de los ciudadanos tanto en la democracia como en la justicia. La solidaridad así vista por autores como Habermas, Rawls y Van Parijs se constituye en el ligamento social que, frente a la creciente ola de individualidad y desinterés por lo público, vuelve a poner a los seres humanos en un dialogo, no solo consigo mismos, sino con su entorno.

Es extraño que la solidaridad durante mucho tiempo se haya manifestado como un discurso secundario que no despertaba inquietud alguna para ser abordada como tema central y, no obstante, surja en estos tiempos de predominio democrático como una condición esencial para la convivencia humana y la armonía social. El discurso solidario hoy crea tendencias en la economía, la política y la filosofía, que ya no son simples discursos al margen, sino que se muestran como dominantes y van más allá de las sencillas relaciones humanas, buscando la conservación del medio ambiente y la creación de unos lazos de armonía con otros seres vivos que habitan con nosotros este planeta. La solidaridad, hoy más que nunca, se torna importante y es fundamental abordarla, estudiarla, darle al discurso que la contiene toda la difusión necesaria, a través de las tendencias económicas, políticas y filosóficas; pero, más importante aún que su difusión en este tiempo es realmente esencial entender los motivos por los cuales actualmente ella 
tiene tanta trascendencia. También es pertinente que, en medio de ese ambiente general sobre el que se difunde el pensamiento solidario, se logre alcanzar cierta unidad conceptual en torno al significado que tiene la solidaridad para los pensadores que en el presente la abordan.

\section{Conclusiones}

La relevancia adquirida por la solidaridad hoy en día es fruto de una disociación entre sociedad civil y Estado, que se agudiza por el sistema-mundo de producción predominante; por ello, autores como Rawl, Habermas, Van Parijs, Lyotar y Levinas la mencionan como un elemento valioso para las sociedades democráticas.

En los pensadores que se han mencionado, se trata la solidaridad de manera más explícita que como fue abordada por los pensadores antiguos. Claro está que, al hacer el recorrido en pensadores antiguos y comparar sus preocupaciones, de las cuales brota su pensamiento, con las de los pensadores actuales, se puede encontrar una explicación de por qué hoy la solidaridad tiene tanta importancia para una sociedad en la que la visión general de igualdad se rompe y la poca credibilidad de los órdenes democráticos genera apatía, en sus ciudadanos, por la participación en todo aquello que tenga que ver con lo público. Al parecer, el momento en el que se vive y en el cual se piensa la solidaridad es la manifestación extrema de lo que Constant llamaba la libertad de los modernos, que consiste en encontrar la libertad en la privacidad del actuar individual, pues al moderno no le interesan los asuntos públicos ni las decisiones que el Estado tome con respecto a diversas situaciones; lo único que quiere es cumplir las obligaciones necesarias con el Estado para que, en su esfera privada, pueda disfrutar tranquilamente de su libertad (Constant, 1819).

Si bien la preocupación de los modernos consiste en encerrarse en su esfera privada para no ser molestados, el problema que surge de ahí, y por el cual los griegos no sufrían, es cómo hacer posible que ese Estado adquiera legitimidad y no se atomice, no se desarticule. Esa preocupación que para los pensadores antiguos no era un problema presente es para los modernos la principal causa de reflexión, motivo por el cual le dedican sus mayores esfuerzos, con el fin hacer posible que se legitimen el orden social en el que se vive, el Estado y la justicia que ahí se imparte. Es por ello que, en pensadores como Rawls, Habermas, Van Parijs, Lyotar y Levinas, la preocupación por la solidaridad se convierte en piso de soporte de sus reflexiones. Es en las obras de estos autores en las cuales se puede encontrar los motivos por los que la solidaridad adquiere importancia hoy en día, no solo como un tema de discusión filosófica académica, sino como reflexión sobre un 
problema objetivo, para el cual la solidaridad repercute como soporte de un "resurgimiento de la sociedad civil".

Finalmente, el núcleo genuino del "resurgimiento de la sociedad civil" al que se refería Boaventura de Sousa está profundamente relacionado con la solidaridad. La reafirmación de los valores de autogobierno, de la expansión de la subjetividad, del comunitarismo y de la organización autónoma tanto de los intereses como de los modos de vida se encuentra inmersa en las reflexiones que se dan en torno a la solidaridad. Por ejemplo: el otro en Levinas, categoría que se encuentra muy ligada a la solidaridad, contempla la expansión de la subjetividad; la democracia deliberativa de Habermas reafirma los valores del autogobierno, y la noción de justicia en Rawls pregona una organización autónoma de los intereses y de los modos de vida, pero basada ya no en la igualdad, sino, en la diferencia. Sin embargo, este "resurgimiento de la sociedad civil" no solo está determinado por una suerte de regreso del actor (Touraine, 1987); la contraparte, es decir, el Estado, también puede permitir que se produzca esto. Tal es el caso de la renta básica de Van Parijs, con la cual la sociedad civil resurge, a partir de la satisfacción de las necesidades materiales, lo que involucra una nueva ruptura de la dicotomía sociedad civil/Estado.

\section{Referencias}

Aristóteles. (1973). La política. Barcelona: Editorial Bruguera.

Cole. G. (1957). Historia del pensamiento socialista I: Los precursores. México: Fondo de Cultura Económica.

Constant, B. (1819). La libertad de los antiguos comparada con la libertad de los modernos. Madrid: Editorial Taurus.

Bobbio, N. (1989). Estado, gobierno y sociedad: por una Teoría General de la Política. México: Fondo de Cultura Económica.

Habermas, J. (1998). Teoría de la acción comunicativa, volumen I. Madrid: Editorial Taurus.

Honneth, A. (1997). La lucha por el reconocimiento: La gramática moral de los conflictos sociales. Barcelona: Editorial Crítica.

Levinas, M. (1998). La huella del otro. México: Editorial Taurus.

Lyotar, J. (2000). La condición posmoderna. Madrid: Editorial Cátedra. 
Marx, K. y Engels, F. (1968). La ideología alemana. Montevideo: Editorial Pueblos Unidos.

Mill, J. (1859). Ensayo sobre la libertad. Madrid: Editorial Mestas.

Moro, T. (1973). Utopía. Barcelona: Editorial Bruguera.

Pardo, I. J. (1990). Fuegos Bajo el Agua: La invención de utopía. Perú: Editorial Ayacucho.

Platón. (2003). Diálogos, tomos I y II. México: Editorial Porrúa.

Rawls, J. (1971). Teoría de la Justicia. México: Fondo de Cultura Económica

Salvat, P. (2007). Teoría de la justicia y solidarismo liberal en John Rawls. En M. Figueroa y D. Michelini (Comps.), Filosofía y Solidaridad. Chile: Universidad Alberto Hurtado.

Sousa Santos, B. (1998). De la mano de Alicia. Lo social y lo político en la posmodernidad. Bogotá: Ediciones Uniandes.

Touraine, A. (1987). El regreso del actor. Buenos Aires: Eudeba.

Van Parijs, P. (2004). Cultural Diversity versus Economic Solidarity. Newyork: Editorial Berg.

Young, M. (2000). La justicia y la política de la diferencia. Madrid: Ediciones Cátedra.

Werner, C. (1966). La filosofía griega. Barcelona: Editorial Labor. 